\title{
A high accuracy method for the simulation of non-ideal optical cavities
}

\author{
Jean-Yves Vinet $\left({ }^{1}\right)$, Patrice Hello $\left({ }^{1,2}\right)$, Catherine N. Man $\left({ }^{2}\right)$ and Alain Brillet $\left({ }^{2}\right)$ \\ (1) Groupe de Recherche sur les Ondes de Gravitation, Laboratoire d'Optique Appliquée, Ecole \\ Polytechnique - ENSTA, 91120 Palaiseau, France \\ ${ }^{2}{ }^{2}$ Groupe Virgo, Laboratoire de l'Accélérateur Linéaire, Université de Paris-Sud, Bâti- \\ ment 208, 91405 Orsay, France
}

(Received 17 October 1991, accepted in final form 18 February 1992)

\begin{abstract}
We present an algorithm able to represent with a high accuracy any kind of stable cavity, even when many static or dynamical defects are present, like misalignments, curvature errors, surface irregularities, substrate inhomogeneities... We first present the theory, giving ideas on its validity domain, and a discussion of its accuracy in terms of a RMS phase error, which is found to be negligible compared to the phase noise due to roughness of optical surfaces. Then we show that the well-known features of ideal resonant cavities are found by the algorithm with a good accuracy. This tool can help for designing laser cavities, mode cleaners, or passive FabryPerot standards ; as an example, some results are presented concerning the design of a very long cavity planned for interferometric purposes.
\end{abstract}

\section{Introduction.}

In the development of new laser configurations, design of passive cavities, information is often needed about tolerances on various optical or photometric parameters such as curvature radii of mirrors, diffraction losses, misalignments, aberrations, bulk index homogeneity of thick optical substrates. Quantitative studies of the behavior of the whole system in the presence of thermal exchanges between the optical beam and the optical elements, or dynamical response to mechanical excitation of these, are sometimes also necessary. A general method of simulation of the optical state of a cavity in the presence of any kind of perturbation is therefore of great use.

Search for specifications of optical components for a very long cavity storing a high power light beam led us to develop a specific numerical tool, able to model weak departure of parameters from nominal values, and to evaluate effects caused by surface distortions even smaller than $\lambda / 1000 \mathrm{rms}$.

We started with the paraxial propagation technique due to Sziklas and Siegman [1], based on two-dimensional Fourier Transforms which allows modeling of all types of surface defects and which is easy to use because a number of performing FFT routines are available in all 
supercomputer libraries. The original part of our work was to find procedures for the control of resonance of high finesse stable cavities, and for the representation of optical surfaces.

Previous models, based on the work of Fox and Li [2], concern modeling of stable cavities with apertures or finite size mirrors, by various resolution methods of the iterative diffraction integrals, like the Prony method [3, 4], or the use of Laguerre-Gauss mode expansions [5, 6]. The direct consideration of the diffraction integrals in real cavities allows in a few cases [7] the study of defects other than simply the finite size of the mirrors or apertures in the cavity. The real advantage of our study is first the use of FFT in beam propagations, and second the fact that we can consider separately or together many defects on the mirrors.

We have performed calculations for cavities with various combinations of defects or with time varying defects, which are almost impossible to treat otherwise. We also give some practical information about the accuracy and implementation of our numerical method.

\section{Theoretical basis of the propagation method.}

In the present section, we recall the mathematical basis of the propagation by Fourier transforms, and propose a discussion of its domain of validity and accuracy. In the scalar approximation, assuming a monochromatic field of time dependence $\exp (-i \omega t)$, the propagation of light obeys the Helmholtz equation :

$$
\left(\Delta+k^{2}\right) \Psi=0
$$

where the complex wave function $\Psi(x, y, z)$ represents any component of the electromagnetic field, and $k=\omega / c$. Discussion of equation (1) in a fully general situation involving the knowledge of the field on a given surface could start from Kirchhoff's equation. However we are going to deal with waves propagating along the $z$ axis, and vanishing outside a finite region of the $(x, y)$ plane. We shall restrict our consideration to the class of functions $\Psi$ having a finite integral of the squared modulus, even when multiplied by powers of $x$ and $y$, and such functions have two-dimensional Fourier transforms (2D-FT):

$$
\mathcal{F} \Psi(p, q, z)=\int_{\mathrm{R}^{2}} \mathrm{~d} x \mathrm{~d} y \mathrm{e}^{-l p x} \mathrm{e}^{-l q y} \Psi(x, y, z) .
$$

The reverse Fourier transform is written as :

$$
\mathfrak{F}^{-1} \Phi(x, y, z)=\left(\frac{1}{2 \pi}\right)^{2} \int_{\mathrm{R}^{2}} \mathrm{~d} p \mathrm{~d} q \mathrm{e}^{1 p x} \mathrm{e}^{l q y} \Phi(p, q, z)
$$

and, in the Fourier space we restrict again our consideration to the class of functions $\Phi$ having integrable square modulus of the 2D-FT even when multiplied by powers of $p$ and $q$.

Such a class of functions involves for example, perturbations of the eigenmodes of cavities which may be approximated by a finite linear combination of Hermite-Gauss modes. In a given transversal plane $z=z_{0}$, the preceding class of functions has a Hilbertian space structure, with the scalar product:

$$
\left\langle\Psi_{1}, \Psi_{2}\right\rangle=\int \Psi_{1}^{*}(x, y, z) \Psi_{2}(x, y, z) \mathrm{d} x \mathrm{~d} y
$$

and the square modulus :

$$
\|\Psi\|=\langle\Psi, \Psi\rangle^{1 / 2}
$$


2.1 PROPAGATION FROM PLANE TO PLANE. - By taking the 2D-FT of equation (1) we obtain a second order differential equation admitting wave solutions propagating to the left and to the right :

$$
\mathfrak{F} \Psi(p, q, z)=\mathrm{e}^{ \pm i z \sqrt{k^{2}-p^{2}-q^{2}}} A(p, q) .
$$

The function $A(p, q)$ is determined by setting $z=z_{1}$ then $z=z_{2}$ in equation (6), giving the propagation relation between the Fourier data on planes $z=z_{1}$ and $z=z_{2}$.

$$
\mathcal{F} \Psi\left(p, q, z_{2}\right)=\mathrm{e}^{ \pm i\left(z_{2}-z_{1}\right) \sqrt{k^{2}-p^{2}-q^{2}}} \mathfrak{F} \Psi\left(p, q, z_{1}\right) .
$$

The phase factor:

$$
G_{k}(p, q, z)=\mathrm{e}^{ \pm l z} \sqrt{k^{2}-p^{2}-q^{2}}
$$

in the Fourier space will be called the « propagator ». The « + or « - sign corresponds to right or left propagating waves. Note that equation (7) results from the only hypothesis that $\Psi$ has a 2D-FT, and the direction of propagation results from the fact that in relevant cases, when diffraction is well described by the paraxial approximation $\left(p^{2}+q^{2} \ll k^{2}\right), \mathcal{F} \Psi$ vanishes outside the circle $p^{2}+q^{2}=k^{2}$. It is also the basis of the algorithm of Sziklas and Siegman [1], and allows the representation of the wave propagation by a linear operator $P$, mapping the Hilbert space at $z_{1}$ onto the Hilbert space at $z_{2}$.

$$
\mathbf{P}=\mathfrak{F}^{-1} G_{k}\left(z_{2}-z_{1}\right) \mathcal{F} .
$$

This formulation of the propagation problem is very efficient with respect to the methods based on direct integration of Kirchhoff's equation (see Refs [2-7]) because the twodimensional integrations here can be decimated by use of the FFT.

However, propagation from plane to plane is not sufficient for our purposes. In general we need to represent propagation from a curved surface (for instance a mirror) to another curved surface. For this purpose, we proceed in the following way : we split the propagation from a surface $\Sigma$ to a surface $\Sigma^{\prime}$ into three sequences : first the propagation from $\Sigma$ to its tangent transverse plane $\Pi$, then the propagation from $\Pi$ to the tangent plane $\Pi^{\prime}$ of $\Sigma^{\prime}$, and finally the propagation from $\Pi^{\prime}$ to $\Sigma^{\prime}$.

2.2 REPRESENTATION OF OPTICAL SURFACES : SHORT DISTANCE APPROXIMATION. - Propagation from a curved surface to its tangent plane will be represented by a simple non-uniform phase factor. We examine carefully this approximation because it depends both on properties of the wave and on those of the optical surface. Namely, we shall consider waves which are at least roughly matched to the nominal parameters of the cavity, and cavities with mirrors having an almost ideal shape. To be more specific and without serious loss of generality, we shall consider a reference cavity having a plane mirror $M_{0}$ at $z=L$, and a spherical mirror $\mathbf{M}_{1}$ of curvature radius $R$ at $z=0$ with $\alpha=R / L>1$. It is well known that at the wavelength $\lambda$, such a cavity has a fundamental Gaussian mode $\mathrm{TEM}_{00}$ with its waist located on the plane mirror, and a beam waist radius :

$$
\frac{\pi w_{0}^{2}}{\lambda}=L \sqrt{\alpha-1} \text { at } z=L \text { and } \frac{\pi w_{1}^{2}}{\lambda}=L \frac{\alpha}{\sqrt{\alpha-1}} \text { at } z=0 .
$$

In practice, considerations about stability and degeneracies of modes lead to values of $\alpha$ such that $\alpha-1$ is not very small (it means that the spot on the curved mirror is not hundred times larger than the spot on the plane mirror). 
We first discuss propagation from plane $z=0$ to the surface $\Sigma$ of the mirror $\mathrm{M}_{1}$, defined by $z=f(x, y)$ (with $f(0,0)=0$ ); by applying equation (7) we get :

$$
\Psi(x, y, f(x, y))=\frac{1}{4 \pi^{2}} \int \mathrm{d} p \mathrm{~d} q \mathrm{e}^{l p x} \mathrm{e}^{l q y} \mathrm{e}^{l f(x, y) \sqrt{k^{2}-p^{2}-q^{2}}} \mathcal{F} \Psi(p, q, 0) .
$$

If $\Psi$ is close to the fundamental Gaussian mode of the cavity, and if $\gamma$ is a constant for which we may neglect $\exp (-x)$ for every $x>\gamma$, the preceding integral is practically bounded to finite values of $p$ and $q$, namely:

$$
p^{2}+q^{2}<\frac{4 \gamma}{w_{0}^{2}}
$$

and we have therefore:

$$
\frac{p^{2}+q^{2}}{k^{2}}<\frac{\gamma \lambda^{2}}{\pi^{2} w_{0}^{2}}=\frac{\gamma}{\pi \sqrt{\alpha-1}} \frac{\lambda}{L}
$$

furthermore, we can write :

$\sqrt{k^{2}-p^{2}-q^{2}}=k-k u(p, q)$ with $u(p, q)=$

$$
=1-\sqrt{1-\frac{p^{2}+q^{2}}{k^{2}}} \approx \frac{p^{2}+q^{2}}{2 k^{2}}-\frac{3}{8} \frac{\left(p^{2}+q^{2}\right)^{2}}{k^{4}}+.
$$

therefore :

$$
\Psi(x, y, f(x, y))=\mathrm{e}^{i k f(x, v)} \frac{1}{4 \pi^{2}} \int \mathrm{d} p \mathrm{~d} q \mathrm{e}^{i p x} \mathrm{e}^{i q y}[1-i k f(x, y) u(p, q)] \mathcal{F} \Psi(p, q, 0) .
$$

Let us evaluate the error caused by using :

$$
\Psi^{\prime}(x, y, f(x, y))=\mathrm{e}^{i k f(x, y)} \Psi(x, y, 0)
$$

instead of equation (11). We can define an rms phase distortion $\Phi$ by :

$$
\left\|\Psi-\Psi^{\prime}\right\|^{2}=\|\Psi\|^{2}+\left\|\Psi^{\prime}\right\|^{2}-2\left\|\Psi \Psi^{\prime}\right\| \cos \Phi
$$

for the normalized waves $\Psi, \Psi^{\prime}$ and assuming a small distortion, this reduces simply to :

$$
\Phi=\left\|\Psi-\Psi^{\prime}\right\|
$$

The rms phase distortion induced by using the approximate short distance diffraction formula is thus :

$$
\Phi=\left\|i k f(x, y) \mathrm{e}^{i k f(x, y)} \frac{1}{4 \pi^{2}} \int \mathrm{d} p \mathrm{~d} q \mathrm{e}^{i p x} \mathrm{e}^{i q y} u(p, q) \mathfrak{F} \Psi(p, q, 0)\right\| .
$$

The order of magnitude of $\Phi$ can be estimated in the case where the surface $\Sigma$ is nearly parabolic, and the wave $\Psi$ nearly Gaussian : the main contribution to the preceding integral will come from the following terms :

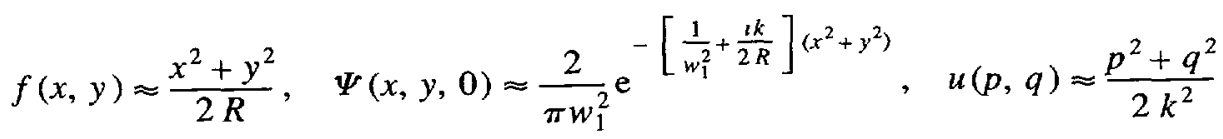


by definition of the phase distortion :

$$
\Phi=\left\|\frac{f(x, y)}{2 k}\left(\partial_{x}^{2}+\partial_{y}^{2}\right) \Psi(x, y, 0)\right\|
$$

which is given by a direct calculation :

$$
\Phi=\frac{1}{4 \pi} \sqrt{\frac{\alpha+5}{\alpha(\alpha-1)^{2}}} \frac{\lambda}{L} .
$$

In the example of a small He-Ne laser, $L=0.2 \mathrm{~m}, \lambda=6.328 \times 10^{-7} \mathrm{~m}, \alpha=1.10$, we have $\Phi \approx 6 \times 10^{-6} \mathrm{rd}$; for the giant cavities in Gravitational Waves Interferometric Detectors $L=3 \times 10^{3} \mathrm{~m}, \lambda=1.06 \times 10^{-6} \mathrm{~m}, \alpha=1.15$, we have $\Phi \approx 4 \times 10^{-10} \mathrm{rd}$; note that a distortion of the optical surfaces of $\lambda / 1000 \mathrm{rms}(1 \mathrm{~nm}$ at $1.06 \mu \mathrm{m})$, is equivalent to a phase distortion of about $6 \times 10^{-4} \mathrm{rd}$. $\Phi$ is thus generally negligible, and we can represent the propagation from a plane to the tangent curved mirror by a simple phase factor. It can be shown by a similar discussion that propagation from a curved mirror to the tangent plane can be represented by a phase factor with the same accuracy :

$$
\Psi(x, y, 0)=\mathrm{e}^{-1 k f(x, y)} \Psi(x, y, f(x, y)) .
$$

Finally, reflection on the curved mirror is equivalent to multiplication by the phase factor

$$
\mathrm{e}^{-2 \imath k f(x, y)}
$$

We have just seen that the surface of a mirror has the effect of adding to the phase of the wave function a phase related to the surface equation of the mirror. But a mirror, and generally any other optical component, acts also on the amplitude of the wave. Owing to the previous analysis, to each of these we may associate a linear operator acting on the wave complex amplitude. Refraction and reflection are represented by operators of the form :

$$
T(x, y)=t \mathrm{e}^{l f(x, y)} d(x, y) \text { and } R(x, y)=\operatorname{ir} \mathrm{e}^{l g(x, y)} d(x, y)
$$

where $t$ and $r$ are the ordinary scalar amplitude transmission and reflection coefficients and $f$, $g$ two real functions representing the local phase change due to either the reflecting surface shape (for a mirror) or the variable optical thickness (for a thin lens). $d(x, y)$ represents the diaphragm function of the optical element : $d$ is zero outside, and unity inside. Note that a $\pi / 2$ phase lag is assumed for a reflection. For a thick lens, we have first a refraction step, next a propagation step and then a second refraction step.

The functions $f, g$ and $d$ may be the sum of a large class of terms, including the non-ideal geometrical form and various perturbation terms such as : finite size of the mirrors, surface defects, curvature errors, misalignments (transverse displacements and tilts on the optical axis), or inhomogeneity of the substrate index.

The conclusion of this section is that we can represent propagation in the free space by a phase factor in the Fourier space according to equation (7), and the reflection or the refraction on curved surfaces by phase factors in the direct space. Thus, implementation of the method requires a rectangular grid for sampling of the complex amplitudes and the optical surfaces; the corresponding grid in the Fourier space will be used for sampling the transformed amplitudes and the propagator, a 2D-FFT subroutine, and a fast procedure for multiplying two complex arrays. These requirements are especially suitable for vector computers.

Let us now turn our attention to the specific form of the reflection and refraction operators. 
2.3 OPTICAL OPERATORS. - Consider a mirror with a surface shape like that represented in figure 1 , where $z=f(x, y)$ is the equation of the non-ideal surface of the miror; the other surface is assumed to be a perfect plane laying at $z=0$. The perturbed surface is assumed to have a reflecting coating, while the substrate is assumed to have an index $n$. We have to determine the phase factor corresponding to a transmission through the mirror or a reflection on it.

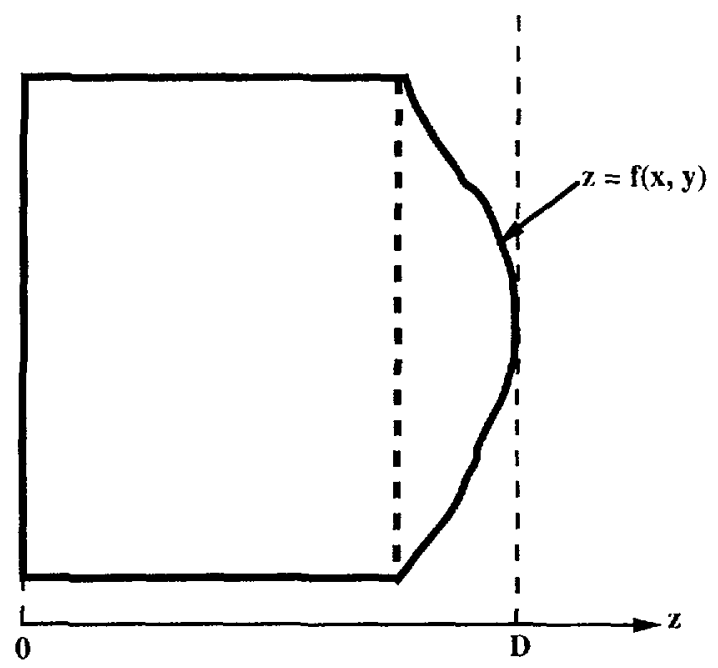

Fig. 1. - Sketch of a mirror with a surface equation $z=f(x, y)$.

First, the light which propagates from the plane $z=0$ to the plane $z=D$, outside the mirror, undergoes an optical path change $\delta_{1}(x, y)=n f(x, y)+(D-f(x, y))$; the first term is for the propagation to the surface of the mirror, and the second is for the propagation from the surface to the plane $z=D$. The same optical path change is seen in a reverse propagation. The refraction operator has the specific form :

$$
T(x, y)=t \mathrm{e}^{i k D} \mathrm{e}^{i k(n-1) f(x, y)} d(x, y) .
$$

Let us now consider a wave propagating from the plane $z=0$, reflected on the coating at the mirror surface and finally propagating back to the plane $z=0$. The optical path change is then $2 n f(x, y)$. The reflection operator for a wave coming from the left of the mirror takes the form

$$
R_{\text {left }}(x, y)=i r \mathrm{e}^{2 i k n f(x, y)} d(x, y)
$$

Finally let us take a wave coming from the right of the mirror, propagating from the plane $z=D$ to the surface $z=f(x, y)$ reflected on the coating and then propagating back to the plane $z=D$. The optical path seen by the light is $D-f(x, y)$ one way, plus $D-f(x, y)$ back, and the reflection operator for the wave coming from the right of the mirror is then :

$$
R_{\mathrm{right}}(x, y)=i r \mathrm{e}^{2 i k D} \mathrm{e}^{-2 i k f(x, y)} d(x, y) .
$$

We see that the operators of reflection must be split into right and left operators, according to the direction of the incident wave. Note also that $D$ can be arbitrarily chosen : for instance, we may take it as the maximum value of the function $f(x, y)$. 
Note that the imperfections of the substrate of the mirrors related to a non-uniform index distribution can be represented by an integrated optical path change $\delta(x, y)$ for the light which passes through the materials ; this means that we consider the imperfect substrate like a phase lens. The corresponding refraction operator is for instance :

$$
T(x, y)=t \mathrm{e}^{i k \delta(x ; y)} d(x, y) .
$$

Thus we know how to write the different optical operators related to a single mirror. We can now take two of them and build a cavity.

\section{Model of resonant cavity.}

In this paragraph, we show how a resonant Fabry-Perot cavity working for instance as a reflector can be represented by a linear operator and the reflected wave can be obtained with a known incoming beam. We consider a cavity of length $L$ illuminated by a wave of amplitude $\psi_{\text {in }}$. We call $\mathrm{T}_{1}$ and $\mathrm{R}_{1}$ the refraction and reflection operators of the input mirror. $\mathrm{R}_{1}$ may be split into left and right parts, according to the previous section, depending on the relevant defects. Let $R_{2}$ be the reflection operator of the end mirror, and $P$ the propagator of the cavity :

$$
\mathbf{P}=\mathfrak{F}^{-1} G_{L} \mathcal{F}
$$

With the help of the notation of figure 2, we get the following relations between the amplitudes of the light in the cavity, before and after the interaction with the mirrors :

$$
\left\{\begin{array}{l}
\psi_{1}=\mathrm{T}_{1} \psi_{\text {in }}+\mathrm{R}_{1} \psi_{4} \\
\psi_{2}=\mathrm{P} \psi_{1} \\
\psi_{3}=\mathrm{R}_{2} \psi_{2} \\
\psi_{4}=\mathrm{P} \psi_{3} \\
\psi_{\text {out }}=\mathrm{R}_{1} \psi_{\text {in }}+\mathrm{T}_{1} \psi_{4}
\end{array}\right.
$$

where $\psi_{\text {out }}$ is the amplitude of the reflected wave, which is written :

$$
\Psi_{\text {out }}=R_{1} \Psi_{\text {in }}+T_{1} \mathrm{PR}_{2} \mathrm{P} \Psi_{1}
$$

The problem is thus reduced to the determination of the resonant intra-cavity wave $\Psi_{1}$. If we define a cavity operator $\mathrm{C}$, as :

$$
\mathrm{C}=\mathrm{R}_{1} \mathrm{PR}_{2} \mathbf{P} .
$$

It is easily seen that $\Psi_{1}$ obeys the implicit equation :

$$
\psi_{1}=\mathrm{T}_{1} \psi_{\text {in }}+\mathrm{C} \psi_{1}
$$

The main task of the simulation consists in solving this equation, once all the operators have been defined. From the numerical point of view, we start by taking the $\mathrm{TEM}_{00}$ mode of the ideal cavity times the resonance factor as a first estimate, because the correct solution is expected to be close to it. Then we solve equation (34) by iterations until the standard deviation between the last two estimates for the intra-cavity wave amplitude is less than a certain level, which will be called the accuracy of the method and which will be defined below.

Now we turn our attention to the problem of the resonance of a non-ideal Fabry-Perot resonator. The resonance conditions of an ideal cavity are well-known, but when the mirrors 


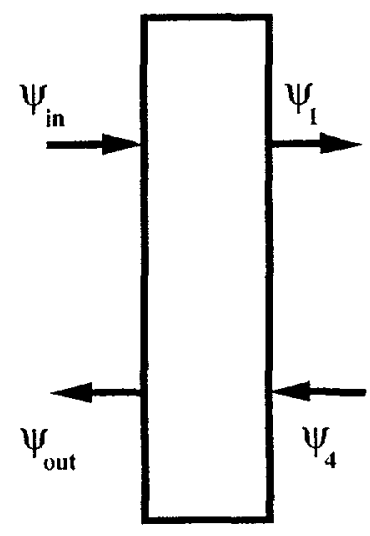

INPUT

MIRROR

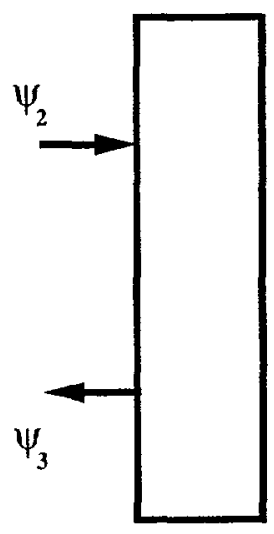

END MIRROR

Fig. 2. - Waves in a cavity : notation.

get strange shapes, or when they are tilted, we have to find the cavity length corresponding to the resonance. Note that tuning the length of the resonator, or the frequency of the source is equivalent to adjusting a uniform supplementary phase in the propagator. Optimization of this phase could be obtained by running many times the numerical code in order to find the maximum of the stored power, but it will be too expensive in terms of computer time. Hopefully, we always consider small defects, because the optics can be assumed to have a fraction of wavelength of imperfections. This gives the idea of a perturbation calculation. Let $\mathrm{C}$ be the cavity operator and let $\varphi$ a uniform phase we add to $\mathrm{C}$; we want to optimize $\varphi$ in order to achieve the fine tuning of the cavity; the intra-cavity wave $\psi_{1}$ obeys the implicit equation :

$$
\psi_{1}=\mathrm{T}_{1} \psi_{\text {in }}+\mathrm{Ce}^{i \varphi} \psi_{1}
$$

Let $\mathrm{C}_{0}$ be the ideal cavity operator corresponding to ideal shapes, without any optical losses. The spectrum of $\mathrm{C}_{0}$ is discrete, and the eigenmodes (for example Hermite-Gauss modes) form a complete orthogonal basis for the space of the light wave amplitudes. Let us note $\Phi_{n}^{(0)}$ the eigenmodes of the ideal cavity, and $\lambda_{n}^{(0)}$ the related eigenvalues, $\Phi_{0}^{(0)}$ being the fundamental $\mathrm{TEM}_{00}$ mode (for the sake of simplicity in the notation, we use a single index, although the Hermite-Gauss or Laguerre-Gauss modes need two indices to be explicitly defined).

We assume that the incoming wave is, in the ideal case, perfectly coupled to one of the eigenmodes of the ideal cavity, for example to the $T E M_{00}$, without loss of generality : $\mathrm{T}_{1} \psi_{\mathrm{in}}$ is then proportional to $\Phi_{0}^{(0)}$.

$$
\mathrm{T}_{1} \psi_{\mathrm{in}}=a \Phi_{0}^{(0)}
$$

then we can write the zero-th order amplitude of the intra-cavity wave $\psi_{1}^{(0)}$ in the ideal case :

$$
\psi_{1}^{(0)}=\frac{a}{1-\lambda_{0}^{(0)} \mathrm{e}^{i \varphi}} \Phi_{0}^{(0)}
$$

which is resonant if $\varphi=-\operatorname{Arg}\left(\lambda_{0}^{(0)}\right)$ : this is the resonance condition for an ideal cavity. 
Consider now the perturbed system : we may expand $\psi_{1}$, at the first order, in a series of the eigenmodes $\Phi_{n}^{(0)}$ (this expansion is unique, as the $\Phi_{n}^{(0)}$ build up a complete orthogonal basis) :

$$
\psi_{1}=A\left[\Phi_{0}^{(0)}+\sum_{p=0}^{\infty} \alpha_{p} \Phi_{p}^{(0)}\right]
$$

where $A$ is an amplitude gain term. The summation runs from zero to infinite (and then includes a component along $\Phi_{0}^{(0)}$ ) in order to make this term appear as a first order correction to the ideal term, which is, according to (37), proportional to $\Phi_{0}^{(0)}$. Furthermore, we can always find a scalar $\lambda$ and a wave $\psi$ so that :

$$
\mathrm{C} \psi_{1}=\lambda \psi_{1}+\psi
$$

with the following orthogonality condition, which definitely gives $\lambda$ and $\psi$ :

$$
\left\langle\psi, \Phi_{0}^{(0)}\right\rangle=0
$$

Equation (35) then becomes :

$$
A\left[\Phi_{0}^{(0)}+\sum_{p=0}^{\infty} \alpha_{p} \Phi_{p}^{(0)}\right]=\mathrm{T}_{1} \psi_{\mathrm{in}}+\lambda \mathrm{e}^{j \varphi} A\left[\Phi_{0}^{(0)}+\sum_{p=0}^{\infty} \alpha_{p} \Phi_{p}^{(0)}\right]+\mathrm{e}^{j \varphi} \psi
$$

and we get by projection this function onto the function $\Phi_{0}^{(0)}$.

$$
A=\frac{\left\langle\mathrm{T}_{1} \psi_{\mathrm{in}}, \Phi_{0}^{(0)}\right\rangle}{1-\lambda \mathrm{e}^{i \varphi}} \frac{1}{1+\alpha_{0}}
$$

The scalar product in equation (42) denotes the coupling between the incoming wave, which excites the cavity, and the eigenmode we want to excite. It means that now it is clear that the value of $\varphi$ which gives the maximum of $A$ (and then the maximum power in the cavity) is :

$$
\varphi=-\operatorname{Arg}(\lambda)
$$

From equations (38), (39) and (40), we get $\lambda$ at first order :

$$
\lambda=\left\langle\mathrm{C} \Phi_{0}^{(0)}, \Phi_{0}^{(0)}\right\rangle
$$

which is very similar to the results of the perturbation calculation applied to Hermitian operators in Quantum Mechanics (although here the cavity operator C is not Hermitian).

The result we obtain is then very simple : in order to tune the cavity at resonance on the $\mathrm{TEM}_{00}$ mode, we just have to add the uniform phase $\varphi$ given by :

$$
\varphi=-\operatorname{Arg}\left(\left\langle\mathrm{C} \Phi_{0}^{(0)}, \Phi_{0}^{(0)}\right\rangle\right)
$$

to the cavity operator phase, or equivalently, we add the uniform phase $\varphi_{\text {prop }}$ given by :

$$
\varphi_{\text {prop }}=-\frac{1}{2} \operatorname{Arg}\left(\left\langle\mathrm{C} \Phi_{0}^{(0)}, \Phi_{0}^{(0)}\right\rangle\right)
$$

to the propagator phase. The factor $1 / 2$ appears in equation (45b) because the propagator appears twice in the cavity operator (see Eq. (33)).

Physically, this locking condition means that the entering beam (assumed to be closed to the ideal $\mathrm{TEM}_{00}$ mode) constructively interferes with the same beam which has performed a 
single round trip in the cavity. This constructive interference will assure an optimal increase of the power in the cavity, as the « mode » builds up.

The computation of expression (45) needs only one round trip in the cavity and is therefore very fast. Note that if we want to tune the cavity on another mode, say $\Phi_{n}^{(0)}$, the same calculation is valid, except that we have to change $\Phi_{0}^{(0)}$ into $\Phi_{n}^{(0)}$.

In the next section, we illustrate the preceding theory by checking the agreement of a numerical model with the classical results about Fabry-Perot resonators, and show that the eigenfrequencies found correspond accurately to the theoretical eigenfrequencies.

\section{Checking the method : numerical results.}

We have performed numerical calculations with a model of a very long cavity, as planned in the French-Italian Virgo Project [8] for the interferometric detection of gravitational waves $[9,10]$. The Virgo cavity is $3 \mathrm{~km}$ long, the input mirror is plane, with a power reflectivity $R_{1}=0.85$, and the end mirror is a spherical high reflectivity one $\left(R_{2} \approx 1\right)$ with $3.45 \mathrm{~km}$ curvature radius. We call ideal a cavity obtained by giving a reflectivity of one to both mirrors, keeping the same geometry.

The numerical computations have been performed on the Siemens VP-200 vector supercomputer of the Centre National de la Recherche Scientifique. In any case, the complete execution of the code (including the definition of the different operators, the calculations of the resonance conditions, of the resonant intra-cavity wave and reflected wave) never exceeds about thirty seconds on the VP-200, whatever the defects of the cavity. The same order of magnitude (a factor of 2 at worst) is found for runs on a Cray 2.

Two sorts of noise must be considered in these experiments :

1) The numerical calculation induces round off errors which result in a phase noise for each optical step (reflection, refraction or propagation) depending on the machine precision and on the sampling rate. All our calculations are performed within the Fortran complex* 16 and real ${ }^{*} 8$ options, which induce a phase noise of about $10^{-16} \mathrm{rad}$.

2) Discrete sampling of the optical waves and other elements on an $n \times n$ grid replaces the linear operator $\mathrm{C}$ (for example) by an $n^{2} \times n^{2}$ matrix, whose eigenvectors are not exactly the discretization of the true continuous modes (an $n^{2} \times n^{2}$ matrix has no more than $n^{2}$ eigenvectors, whereas the ideal cavity has an infinity of modes).

We evaluate this discretization noise by computing, for optimized window sizes, the rms phase distortion of a discretized ideal $\mathrm{TEM}_{00}$ mode after one round trip in the discretized ideal cavity; we find :

$$
\begin{array}{lll}
\Phi=1.3 \times 10^{-4} \mathrm{rad} . & \text { for } & n=64 \\
\Phi=2.6 \times 10^{-7} \mathrm{rad} . & \text { for } & n=128 \\
\Phi=2.5 \times 10^{-7} \mathrm{rad} . & \text { for } & n=256
\end{array}
$$

and we see the advantage to run with $n=128$, rather than $n=64$. Especially, for $n=64$ the discretization noise may be not negligible with respect to small defects that we want however to consider; for example, in section 2.2, we have seen that a geometrical distortion of an optical surface of $\lambda / 1000 \mathrm{rms}$ is equivalent to a phase distortion about $6 \times 10^{-4} \mathrm{rad}$. This discretization noise is the main limit for the accuracy of the numerical code, and we have to be aware of it ; all numerical examples in the following sections have been performed with $n=128$, which implies a very small discretization noise $\left(2.6 \times 10^{-7} \mathrm{rad}\right.$. corresponds to a $4.3 \times 10^{-4} \mathrm{~nm}$ surface distortion, not measurable by the present state of the art). 
4.1 EXCITATION OF TRANSVERSE MODES AND SPECTRUM. - In this first « experiment », we use a mismatched incoming wave (having a tilt angle $\theta$ ) in order to excite a series of HermiteGauss eigenmodes of the ideal cavity [11]. By varying an arbitrary uniform phase $\varphi$ in the propagator, we simulate a continuous tuning of the reference cavity, and the intracavity power exhibits resonance peaks at locations we want to compare with the theoretical assignment. The resonance condition for the $\mathrm{TEM}_{m n}$ mode is, after Kogelnik and Li [12] :

$$
k L-(m+n+1) \operatorname{Arctg}\left(\frac{\lambda L}{\pi w_{0}^{2}}\right)+\frac{\pi}{2}=(q+1) \pi
$$

where $q$ is an arbitrary integer. For the arbitrary phase of the propagator, this locates the resonances at :

$$
\varphi(m, n)=(m+n) \operatorname{Arctg}\left(\frac{\lambda L}{\pi w_{0}^{2}}\right) \text { modulo } \pi
$$

the phase offset being chosen at a resonance of the fundamental mode. Figure $3 \mathrm{a}$ is a plot of the intracavity power versus $\varphi$. Vertical bars show the theoretical eigenphases $\varphi(m, n)$. The error in location corresponds to a $10^{-3} \mathrm{~Hz}$ detuning which gives an idea of the accuracy of the method. We found it useful to give the shape of the intracavity wave at some special resonances (see Fig. 4). Note that the reference cavity having mirrors of non unity reflectivities, the spectrum is continuous, which means that at each frequency, the intracavity wave is a mixing of Hermite-Gauss modes. For instance, the first resonance is mainly $\mathrm{TEM}_{10}$ but also contains small amounts of $\mathrm{TEM}_{00}, \mathrm{TEM}_{20} \ldots$ This is why the geometry of this mode is only almost that of the $\operatorname{TEM}_{10}$.

In a similar way, we are able to excite the first transverse Laguerre-Gauss modes, denoted by $\mathrm{TEM}_{p \ell}$ where $p$ is the radial order and $\ell$ the angular order : for example, we illuminate the cavity with a TEM $_{00}$ wave having a waist not perfectly matched to the cavity ; thus we obtain all the series of the axisymmetrical Laguerre-Gauss modes (the non axisymmetrical LaguerreGauss modes are non excited with such an incoming beam). In figure $3 \mathrm{~b}$ we see that the peaks of the numerical resonances are well fitted to the theoretical peaks, which are obtained in the case of Laguerre-Gauss modes from the resonance phases :

$$
\varphi(p, \ell)=(2 p+\ell) \operatorname{Arctg}\left(\frac{\lambda L}{\pi w_{0}^{2}}\right) \text { modulo } \pi .
$$

4.2 STUDY OF DEFECTS. - We now describe a scheme for the analysis and the evaluation of the intrinsic defects of the cavity and give some examples. In each case, we launch the matched $\mathrm{TEM}_{00}$ Gaussian wave $\Psi_{\text {in }}$, and let the reflected wave $\Psi_{\text {out }}$ interfere with $\Psi_{\text {in }}$. The interference involves an arbitrary phase lag which is chosen to minimize $\left\|\Psi_{\text {out }}-\Psi_{\text {tn }}\right\|$. This minimum value of $\left\|\Psi_{\text {out }}-\Psi_{\text {in }}\right\|$ is the rms global phase distortion, recalling equation (18); it has the mean of a measure of a geometry deviation from the perfect $\mathrm{TEM}_{00}$ mode.

In each case we give the intensity distribution $\left|\Psi_{\text {out }}(x, y)-\Psi_{\text {1n }}(x, y)\right|^{2}$ which corresponds to the dark fringe pattern of interference.

4.2.1 Scaling the sizes of the mirrors. - The ideal cavity has infinitely large mirrors. The first perturbation to be studied is the finite size of the mirrors $: 5 \mathrm{~cm}$ radius for the input mirror and $14 \mathrm{~cm}$ radius for the end mirror. This means that the radii exceed by a factor 2.5 the beam 

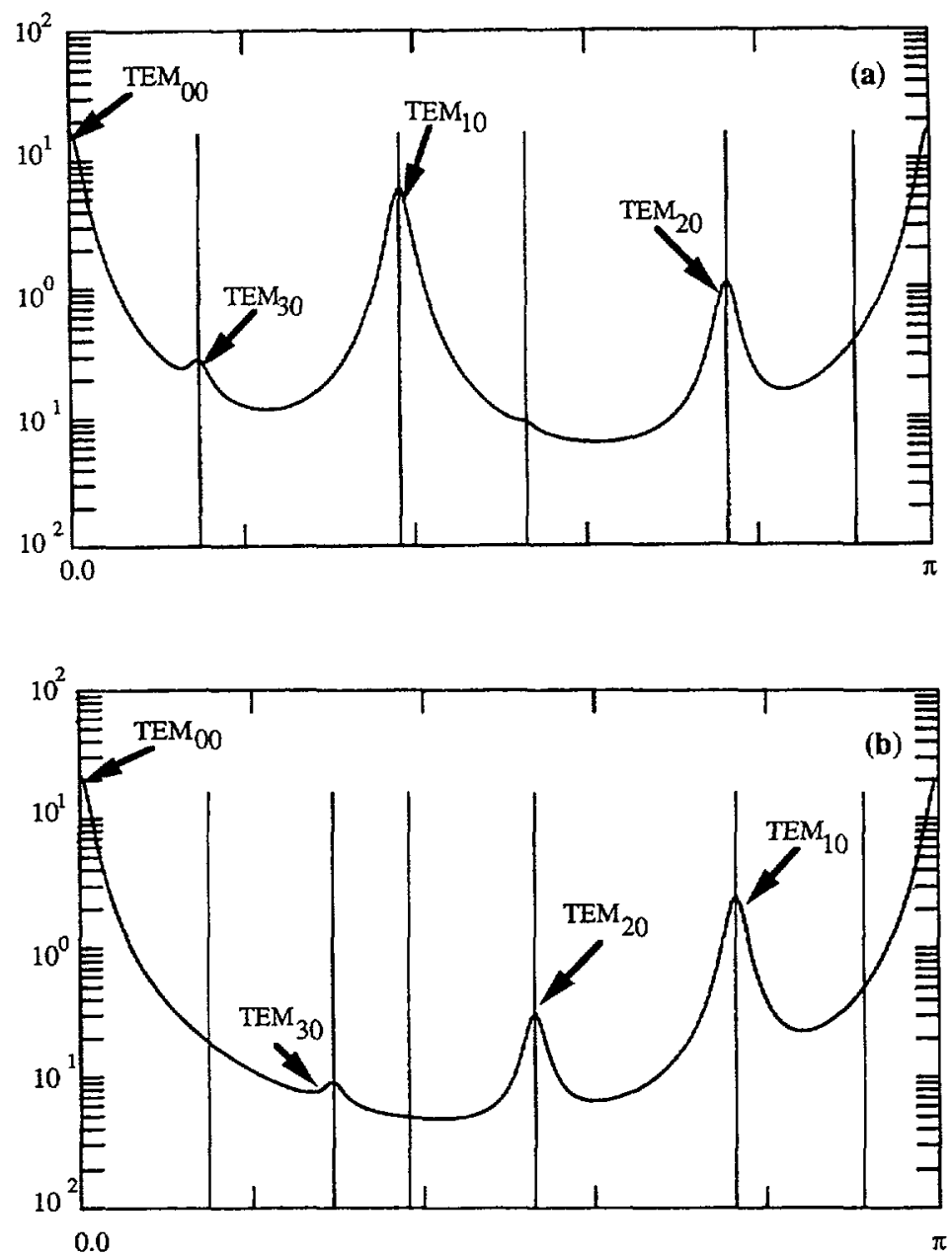

Fig. 3. - Spectrum of a cavity. a) Spectrum obtained by illuminating the cavity with a tilted laser beam; the corresponding Hermite-Gauss modes are excited. b) Spectrum obtained by illuminating the cavity by a laser beam with an unmatched waist : the axisymmetrical Laguerre-Gauss modes are excited.

sizes on the mirrors. The interference pattern between the beam reflected by the cavity and the perfect $\mathrm{TEM}_{00}$ mode is shown in figure 5.

We note that we find the characteristic ring pattern due to the well known coupling of the fundamental mode with higher order axisymmetrical Laguerre-Gauss modes. The rms phase distortion with respect to the ideal TEM $_{00}$ wave is $3.1 \times 10^{-3} \mathrm{rad}$.

4.2.2 Angular misalignments. - In this example we have tilted the end spherical mirror of the cavity by an angle equal to $0.1 \mu \mathrm{rad}$, which corresponds to $0.3 \mathrm{~mm}$ seen over the $3 \mathrm{~km}$ length of the cavity. The interference pattern between the reflected wave and the TEM $_{00}$ mode is shown in figure 6.

We may recognize the interference figure between two pure Gaussian waves but with different propagation directions. This may also be interpreted by the coupling of the TEM $\mathrm{TH}_{00}$ mode of the cavity with higher order Hermite-Gauss modes, mainly TEM $_{10}$ as shown by the figure. The phase distortion is $3.5 \times 10^{-2} \mathrm{rad}$. 


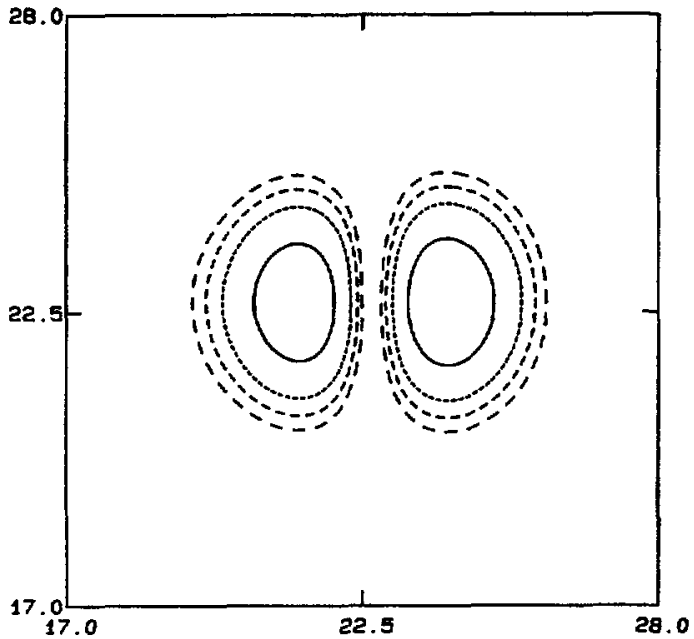

a)

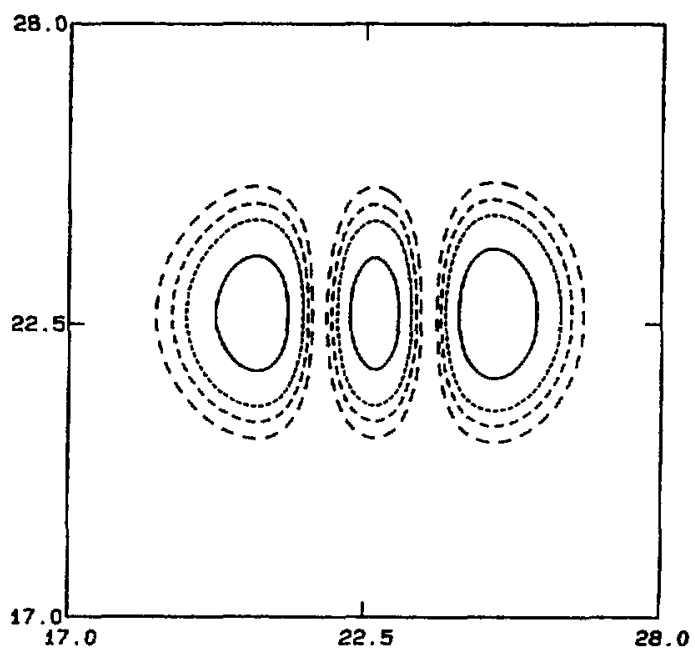

b)

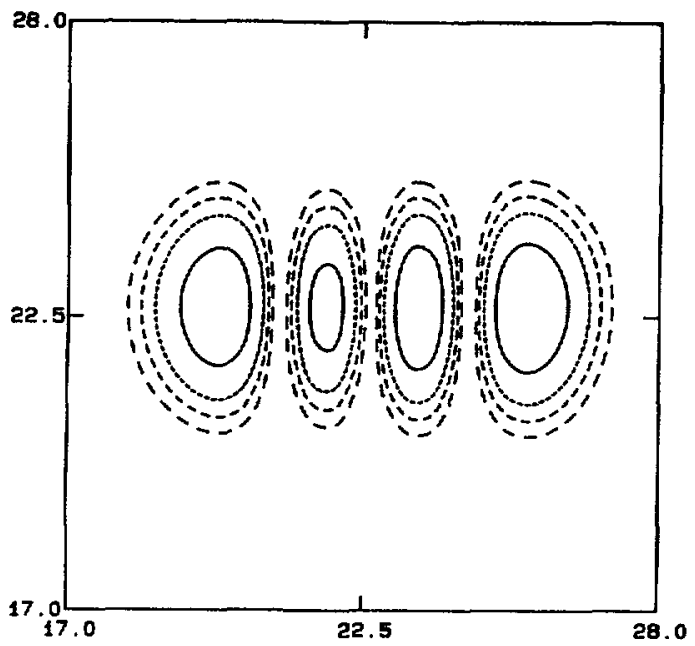

c)

Fig. 4. - View of the different modes excited by the previous methods. Fig. $a, b$ and $c$ show the Hermite-Gauss $\mathrm{TEM}_{10}, \mathrm{TEM}_{20}$ and $\mathrm{TEM}_{30}$ modes, respectively. The intensity isocontours corresponding to the lines: ( respectively. The modes are not perfect because the cavity has some «losses " (reflectivity of the input mirror), so there is in fact a mixture, which results from the coupling of transverse modes to the fundamental TEM $_{00}$ mode : what we call a TEM ${ }_{10}$ mode is in fact a mixture mainly of $\mathrm{TEM}_{00}$ and $\mathrm{TEM}_{10}$ (and also higher modes).

4.2.3 Curvature error. - In this example, the end mirror of the cavity has not its nominal curvature radius $(3.45 \mathrm{~km})$. The relative curvature error is $1 \%$ and is of the order of magnitude of the accuracy announced by most manufacturers. The interference pattern between the reflected beam and the perfect $\mathrm{TEM}_{00}$ is shown in figure 7 .

One can recognize the interference figure between two pure Gaussian waves but with different curvature radii. This may also be interpreted by the coupling of the $\mathrm{TEM}_{00}$ mode of 


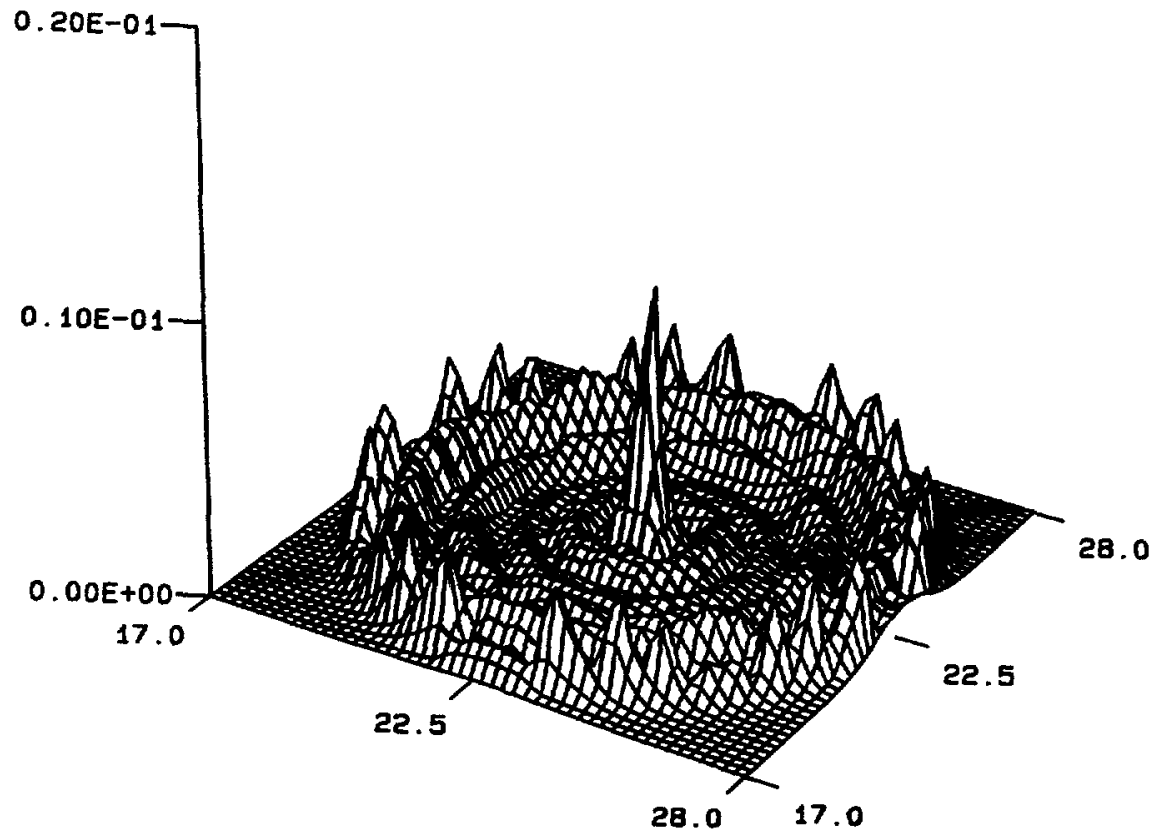

Fig. 5. - Interference pattern from a perfect $\mathrm{TEM}_{00}$ and the beam reflected by the cavity in the case where mirrors have finite sizes. For this chart and all the following, the horizontal scales are expressed in $\mathrm{cm}$ and the vertical scale in $\mathrm{W} \mathrm{m}^{-2}$ Note that a numerical window of $45 \mathrm{~cm}$ has been used.

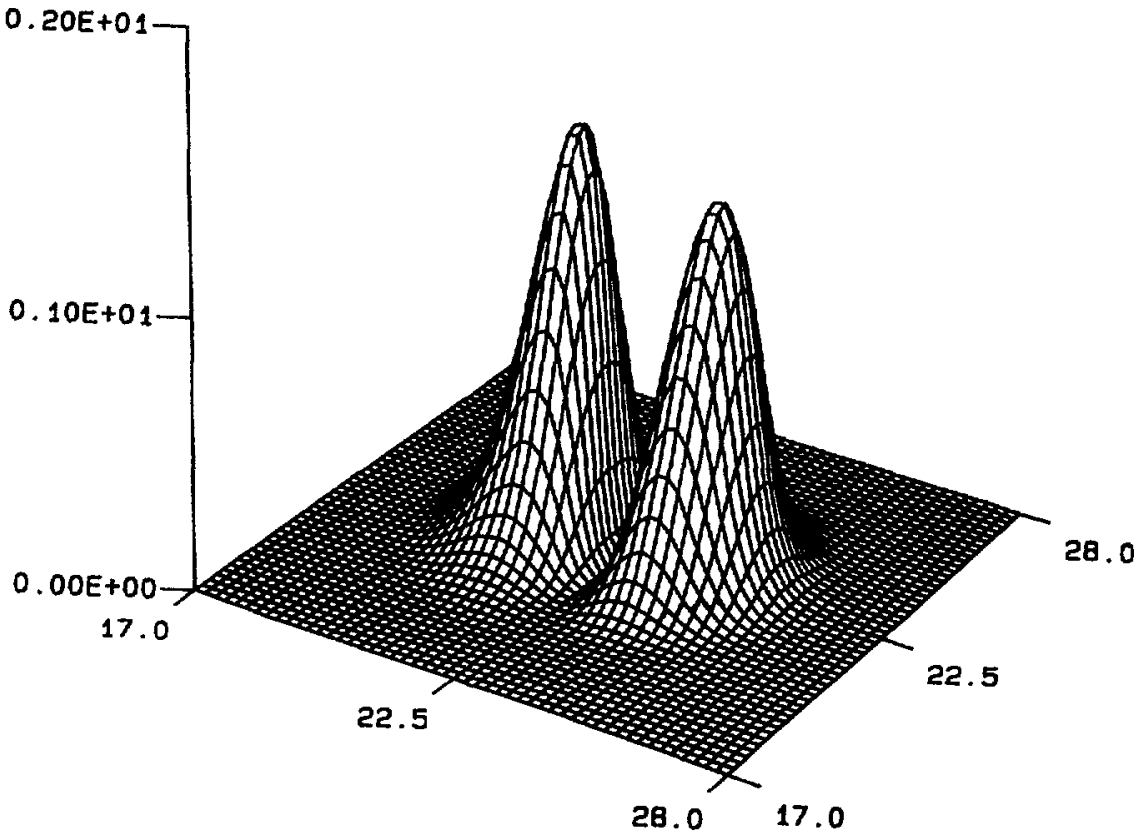

Fig. 6. - Interference pattern from a perfect $\mathrm{TEM}_{00}$ and the beam reflected by the cavity in the case of an angular tilt of the end mirror. 


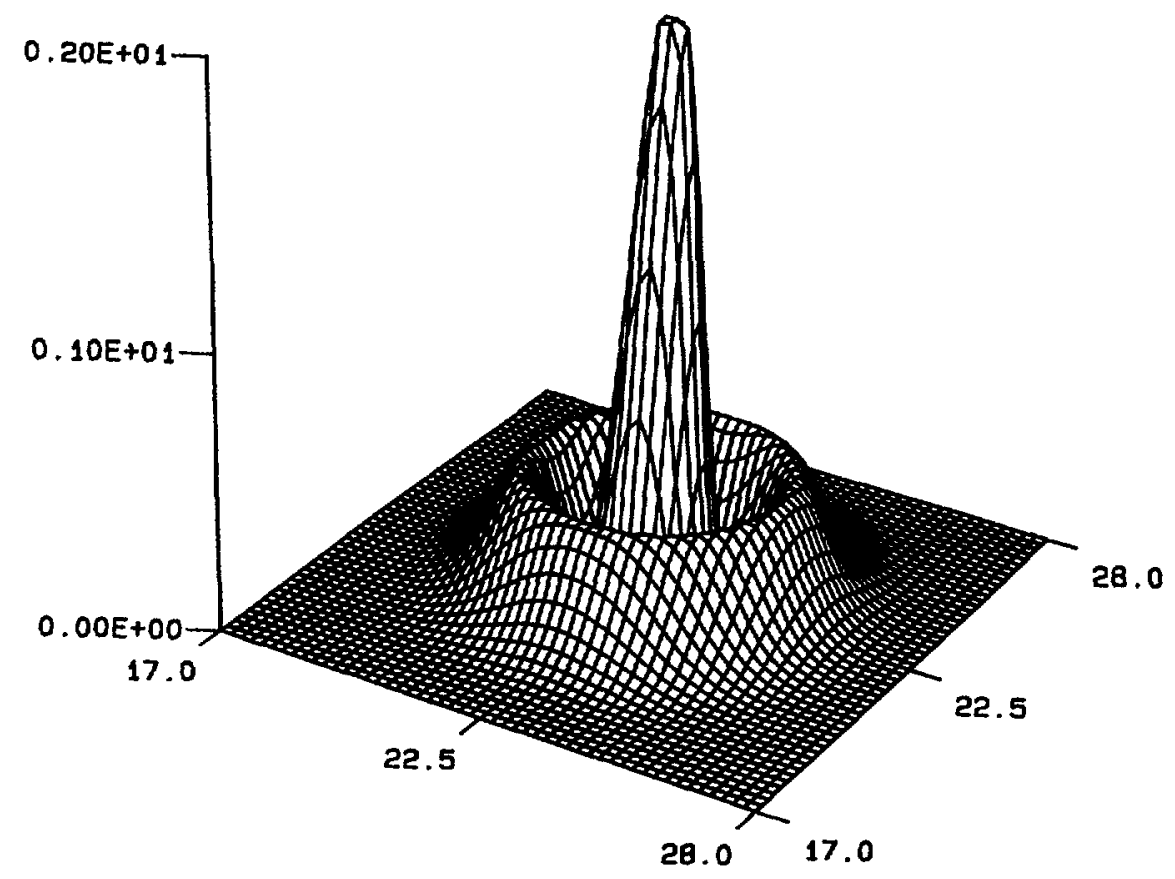

Fig. 7. - Interference pattern from a perfect TEM $_{00}$ and the beam reflected by the cavity in the case of a curvature error of the spherical end mirror of the cavity.

the cavity with higher order axisymmetrical Laguerre-Gauss modes, mainly TEM $_{10}$ as shown by the interference pattern. The resulting phase distortion is $3.7 \times 10^{-2} \mathrm{rad}$.

4.2.4 Surface defects. - This last example concerns the surface defects for the input mirror. The ideal input mirror is a plane mirror, and the distorted surface is shown in figure 8 , the rms value of the aberration being $\lambda / 60$. The related interference pattern between the beam reflected by the cavity and the $\mathrm{TEM}_{00}$ mode of the corresponding ideal cavity is shown in figure 9 , giving a phase distortion of $2.4 \times 10^{-2} \mathrm{rad}$.

\section{Conclusion.}

The presented simulation tool for resonant cavities allows accurate studies of the effects of various static defects and can be employed for optimization of resonators. Because of its time sparing conception, the code can be used in dynamical simulations in which it must be run at each time step with updating of parameters. This makes it possible to study non linear problems such as the detuning of the cavity by thermoelastic deformation of the mirrors heated by the beam [13]. The complete study of thermal effects in passive cavities will be treated in another paper.

The numerical code that we have built has been intensively used in order to determine the specifications of the optics involved in the Virgo Project of a Gravitational Wave Antenna. The results show that the specifications are very stringent and do not exist simultaneously on the same optics yet. This also shows that the code can deal with very weak defects, and confirms that the noise of the method is much below the weakest defects that we have considered in our studies, which are for instance about $\lambda / 1000 \mathrm{mms}$ for the surface quality of the mirrors. 


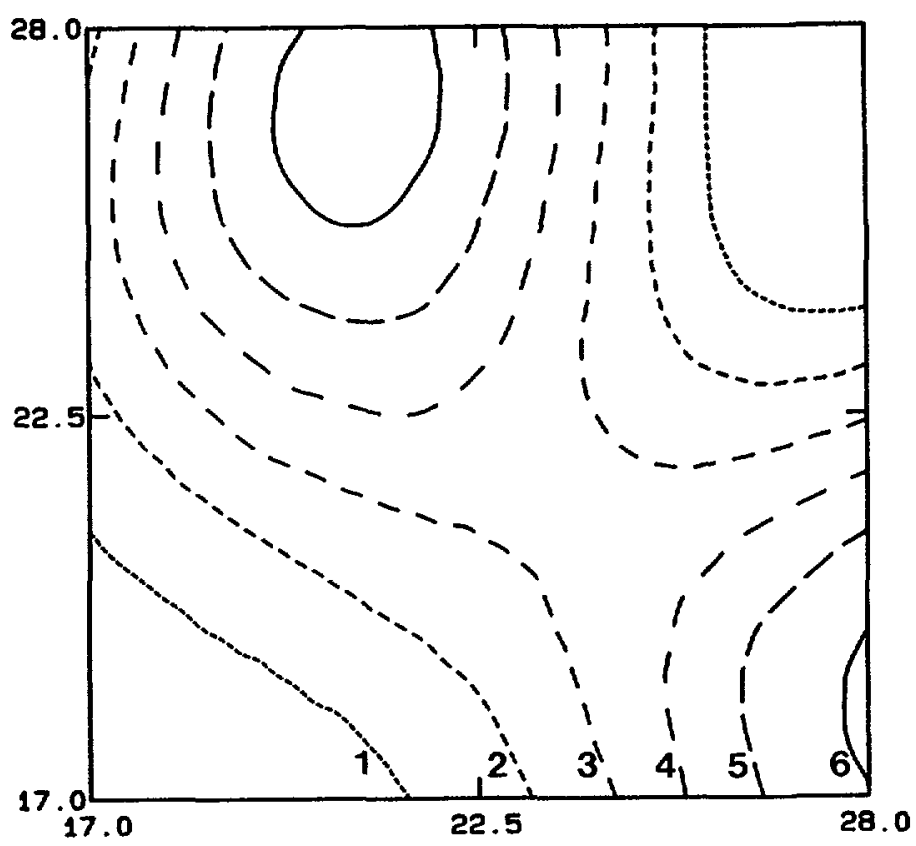

Fig. 8. - View of the surface defects considered for the input mirror. The isocurves numbered from 1 to 6 correspond to respective amplitudes of $5.5 \times 10^{-2} \mu \mathrm{m}, 6.0 \times 10^{-2} \mu \mathrm{m}, 6.5 \times 10^{-2} \mu \mathrm{m}, 7.0 \times 10^{-2} \mu \mathrm{m}$, $7.5 \times 10^{-2} \mu \mathrm{m}, 8.0 \times 10^{-2} \mu \mathrm{m}$ and $8.5 \times 10^{-2} \mu \mathrm{m}$.

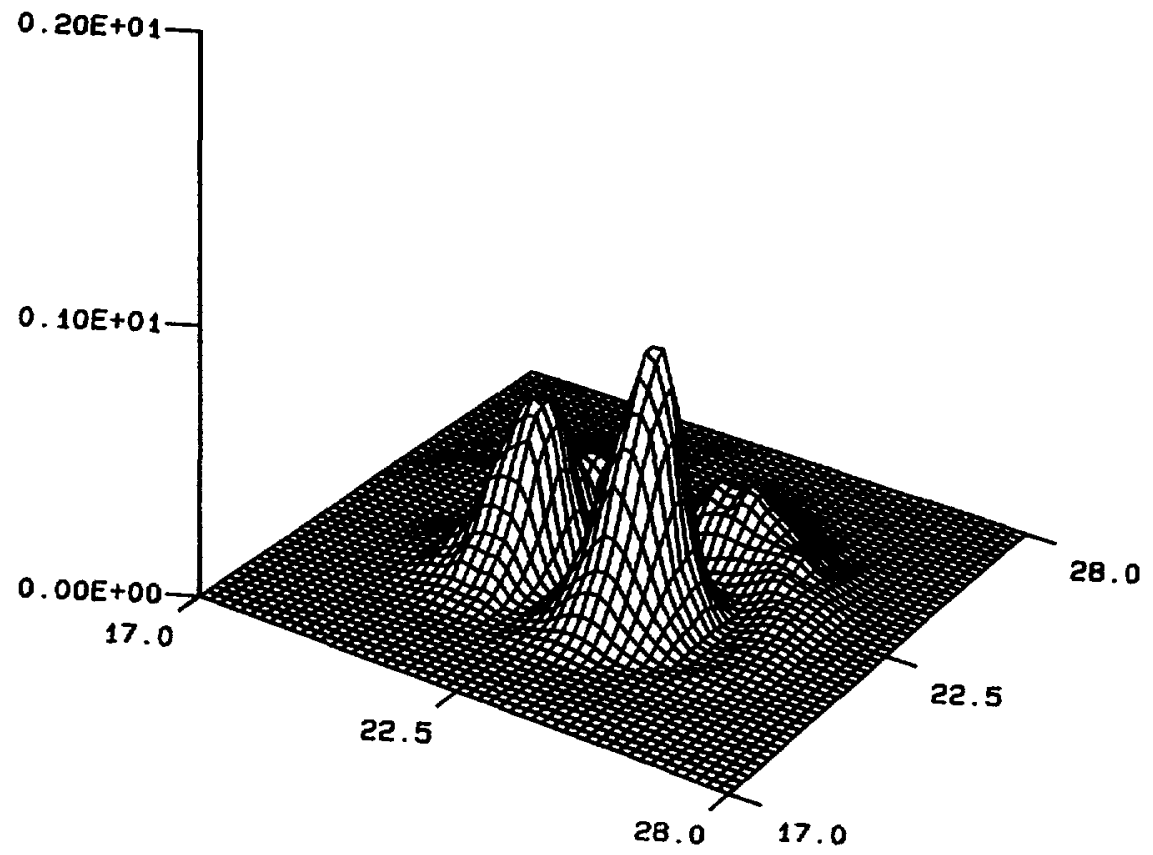

Fig. 9. - Interference pattern from a perfect $\mathrm{TEM}_{00}$ and the beam reflected by the cavity in the case of surface defects of the input mirror of the cavity. The interference has been computed with the surface shape seen in figure 8. 


\section{References}

[1] SzIKLAS E. A. and Siegman A. E., Mode calculations in unstable resonators with flowing saturable gain : Fast Fourier method, Appl. Opt. 14 (1975) 1874.

[2] Fox A. G. and LI T., Computation of optical resonators modes by the method of resonance excitation, IEEE J. Quant. Electron. QE-4 (1968) 460.

[3] SIEgman A. E. and MiLlER H. Y., Unstable resonator loss calculations using the Prony method, Appl. Opt. 9 (1970) 2729.

[4] Piche M., Lavigne P., Martin F. and Belanger P. A., Modes of resonators with internal apertures, Appl. Opt. 22 (1983) 1999.

[5] Kellou A. and StePhan G., Etude du champ proche d'un laser diaphragmmé, Appl. Opt. 26 (1987) 76.

[6] WANG L. Yu and STEPHAN G., Transverse modes of an apertured laser, Appl. Opt. 30 (1991) 1899.

[7] RazumovskiI N. A., Effect of irregularities of the mirrors on stimulated oscillators in a open cavity, Opt. Spectrosc. USSR 67 (1989) 410.

[8] Brillet A., Giazotto A. et al., Virgo Proposal for the construction of a large interferometric detector of gravitational waves (1989, revised 1990) unpublished.

[9] Giazotto A., Interferometric detection of gravitational waves, Phys. Rep. 182 (1989) 365.

[10] THORNE K. S., Gravitational Radiation in 300 Years of Gravitation (Cambridge University Press, Cambridge, 1987).

[11] ANDERSON D. Z., Alignment of resonant optical cavities, Appl. Opt. 23 (1984) 2944.

[12] KogeLnik H. and Li T., Laser beams and resonators, Appl. Opt. 5 (1966) 1550.

[13] Hello P. and VINET J.-Y., Modelling of the VIRGO interferometer, Proceedings of the Elizabeth and Frederick White Research Conference on Gravitational Astronomy : Instrument Design and Astrophysical Prospects, D. E. McClelland and H.-A. Bachor Eds. (World Scientific, Singapore, 1991) p. 1-13;

Hello P., Thesis (Paris XI University, 1990) unpublished. 